\title{
Phasic norepinephrine: A neural interrupt signal for unexpected events
}

\author{
PETER DAYAN \& ANGELA J. YU \\ Gatsby Computational Neuroscience Unit, University College London, London, UK \\ (Received 7 April 2006; accepted 11 fuly 2006)
}

\begin{abstract}
Extensive animal studies indicate that the neuromodulator norepinephrine plays an important role in specific aspects of vigilance, attention and learning, putatively serving as a neural interrupt or reset function. The activity of norepinephrine-releasing neurons in the locus coeruleus during attentional tasks is modulated not only by the animal's level of engagement and the sensory inputs, but also by temporally rich aspects of internal decision-making processes. Here, we propose that it is unexpected changes in the world within the context of a task that activate the noradrenergic interrupt signal. We quantify this idea in a Bayesian model of a well-studied visual discrimination task, demonstrating that the model captures a rich repertoire of noradrenergic responses at the sub-second temporal resolution.
\end{abstract}

Keywords: Noradrenaline, norepinephrine, interrupt, locus coeruleus

\section{Introduction}

Neuromodulators have long attracted empirical and theoretical attention due to their cerebral ubiquity, evolutionary longevity and temporal complexity. In particular, they have distinct activity profiles and implied functional roles over many different time-scales, from milliseconds to hours (e.g., Marder \& Thirumalai 2002). There are various general notions about their roles, such as the regulation of sleeping and waking (Jouvet 1969), and the modulation of signal to noise ratios of cortical neurons, a classical idea (Foote et al. 1975; Rolls 1984; Mantz 1988) that has been remarkably persistent (e.g., Nicola et al. 2004). However, these are slowly giving way to more specific computational ideas (Servan-Schreiber et al. 1990; Montague et al. 1996; Usher et al. 1999; Dayan \& Yu 2001; 2003; Doya 2002; Yu \& Dayan 2002; 2003; 2005; Daw et al. 2002; Brown 2004; Brown 2005; Aston-Jones \& Cohen 2005a;b; Bouret \& Sara 2005), based on such notions as optimal gain scheduling, prediction error, uncertainty and reset.

In this paper we concentrate on the neuromodulator norepinephrine (NE; also known as noradrenaline), whose activity and function have been studied in a variety of tasks that demand vigilance, attention and selective sensory processing (Foote 1980; Aston-Jones \& Bloom 1981; Sara \& Segal 1991; Aston-Jones et al. 1994; Vankov et al. 1995; Aston-Jones et al. 1997; 2000; Witte \& Marrocco 1997; Rajkowski et al. 2004; Bouret \& Sara 2004; 2005).

Figure 1 contains some of the key data that provide a challenge to a comprehensive understanding of NE functions. Previously, we have proposed that medium term NE activation 
A

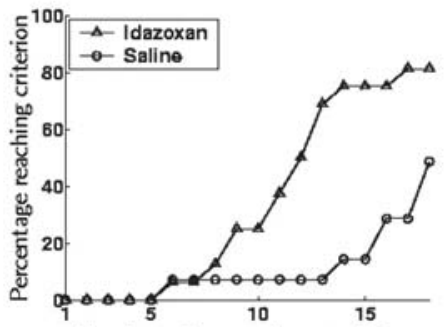

No. days after contingency shift
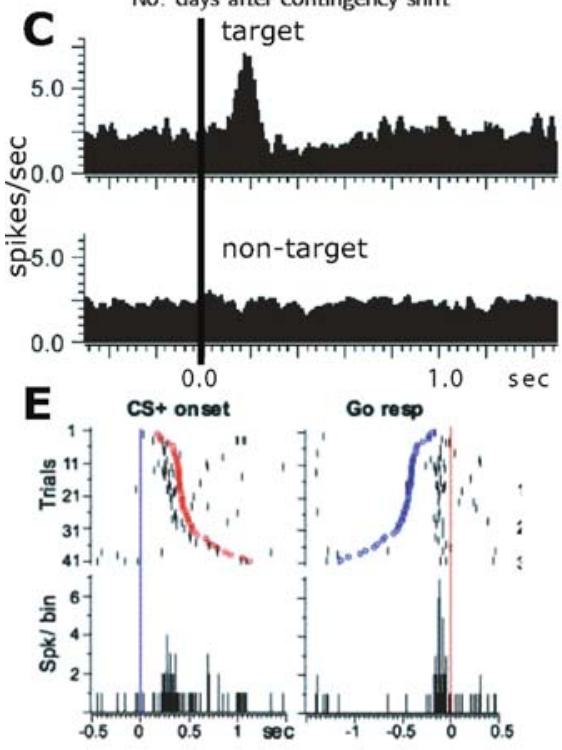

B

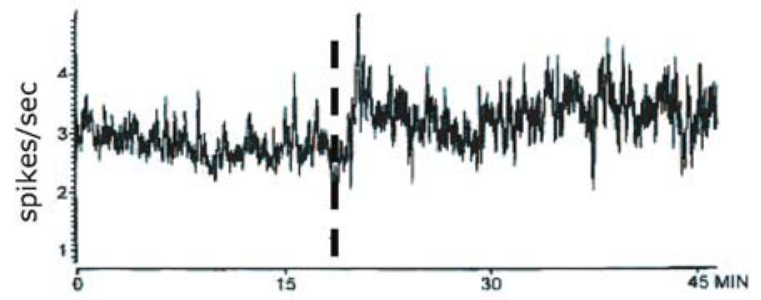

D

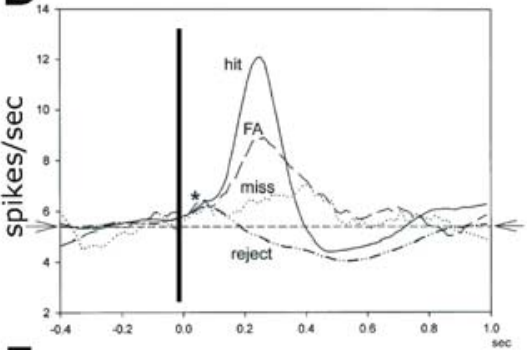

F

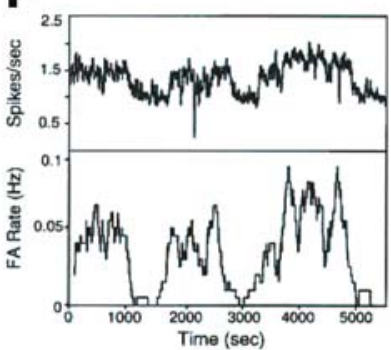

Figure 1. NE activity and effects. A) Rats solved a sequential decision problem with two sets of cues (spatial and visual). When the relevant cues were switched after a few days of learning (from spatial to visual), rats with pharmacologically boosted NE ("idazoxan") learned to use the alternative set of cues faster than the controls. Adapted from Devauges \& Sara (1990). B) Monkeys solved a vigilance task in which they had to react to rare targets and ignore common distractors. The trace shows the activity of a single norepinephrine neuron around the time of a reversal between target and distractor cues (vertical dashed line). The tonic activity is elevated for a considerable period. Adapted from Aston-Jones et al. (1997). C) In the same vigilance task, single NE cells are activated on a phasic time-scale stimulus locked (vertical line) to the target (upper plot) and not the distractor (lower plot). Adapted from Rajkowski et al. (2004). D) Again in the same task, the average responses of a large number of norepinephrine cells (over a total of 41,454 trials) stimulus locked (vertical line) to targets or distractors, sorted by the nature and rectitude of the response. The asterisk marks early activation of the neurons by the stimulus, which is not different for the different types of trial. Adapted from Rajkowski et al. (2004). E) In a GO/NO-GO olfactory discrimination task for rats, single units are activated by the target odor (and not by the distractor odor), but are temporally much more tightly locked to the response (right) than the stimulus (left). Trials are ordered according to the time between stimulus and response, with the red and blue marks showing the time of the response and stimulus respectively. From Bouret \& Sara (2004). F) Correlation between the gross fluctuations in the tonic activity of a single NE neuron (upper) and performance in the simple version of the vigilance task (lower, measured by false alarm rate). From Usher et al. 1999.

(on the order of minutes) is involved in signaling unexpected uncertainty that arises from unpredicted changes in the nature of a task or behavioral context. Figure 1A and B illustrate some of the data that motivated our earlier work. However, Figures $1 \mathrm{C}-\mathrm{F}$ demonstrate that there are significant regularities in the faster timescale activation of $\mathrm{NE}$ neurons that also 
require explaining. Here, we propose the complementary notion that phasic NE also signals unexpected uncertainty, but that arising from unexpected events or state changes within a task. This uncertainty signal in turn interrupts the on-going cognitive processing associated with the default task state.

In the task of Figure 1A, rats are first trained to follow a set of spatial cues to navigate a maze, but then they have to learn to attend to a set of visual cues instead. Following this contingency change, rats whose NE levels have been elevated, via the injection of idazoxan (Curet et al. 1987), adapt to the new task contingencies significantly faster (Devauges \& Sara 1990). The activity of noradrenergic cells has been directly studied in a discriminative vigilance task, in which monkeys must make responses to rare targets and withhold them to common distractors (Aston-Jones et al. 1994; see also Aston-Jones et al. 1997; 2000; Rajkowski et al. 2004). Figure $1 \mathrm{~B}$ shows that the activity is elevated over the medium term following a reversal (vertical dashed line) as to which stimulus is the target and which the distractor. Based on these data, together with other results and ideas on the cellular effects of NE (Hasselmo et al. 1997; Aston-Jones et al. 2000; Berridge \& Waterhouse 2003; Usher et al. 1999), we suggested previously (Yu \& Dayan 2003; 2005) that medium term NE (on the order of minutes) reports unexpected uncertainty arising from unpredicted changes in an environment or task. This signal is a key part of an approximately optimal strategy for strategic adaptation in a non-stationary environment. It can also be seen as a part of a broader hypothesis about the regulation exerted by norepinephrine over exploration (versus exploitation) as a whole (e.g., Usher et al. 1999; Aston-Jones et al. 2000; Doya 2002; Aston-Jones \& Cohen 2005a;b). Drastic changes in the environment induce unexpected model uncertainty (signaled by NE), which, in turn, encourages more exploratory behaviors appropriate for establishing a new model of the external environment. According to our theory, NE operates in collaboration with a putatively cholinergic signal which reports on expected uncertainty, arising from known variability or noise in the current environment.

In contrast to these relatively long-term properties of NE signaling, there is also a host of data on phasic NE responses (e.g., Foote et al. 1980; Aston-Jones \& Bloom 1981; Sara \& Segal 1991; Aston-Jones et al. 1994; 1997; 2000; Vankov et al. 1995; Rajkowski et al. 2004; Bouret \& Sara 2004; Clayton et al. 2004). Some of these results are shown in Figures 1C;D;E. The data in Figure 1C;D come from a version of the discrimination task in Figure 1B (AstonJones et al. 1994; 1997; 2000; Rajkowski et al. 2004): monkeys gain reward by responding appropriately to rare visual targets (rectangles that are oriented in a particular direction), whilst ignoring distractors (rectangles oriented in the perpendicular direction). Under these circumstances, NE is typically activated within less than $200 \mathrm{~ms}$ of the presentation of a target. By comparison, it is weakly activated, if at all, by distractors (Figure 1C). There is also a clear relationship between the NE activity profile and the trial type (hit, miss, false alarm, correct reject; see Figure 1D). There is also some preliminary evidence that the activity is weaker if the targets are more common (Rajkowski et al. 2002; Aston-Jones et al. 1994). In fact, the signal is more tightly related in time to the (subsequent) action than the (preceding) stimulus in a related task (Clayton et al. 2004), as well as in rats performing an olfactory discrimination task (Figure 1E; Bouret \& Sara 2004).

The diverse array of data on NE responses provides both an opportunity and a challenge to a comprehensive theory of NE function. Given that NE is simultaneously released to extravagantly widespread targets (Freedman et al. 1975; Garver \& Sladek 1976; Foote et al. 1983), its potentially powerful effects over diverse neural functions deserve careful analysis. Since this phasic NE signal arises on every trial in an extremely well-learned task with stable stimulus contingencies, it clearly cannot be indicating the sort of unpredicted task changes that were the focus of our previous model (Yu \& Dayan 2003; 2005). Here, we suggest 
that phasic NE plays a complementary role to tonic NE, signaling unexpected uncertainty regarding the state within a task rather than about the task itself.

Our proposal is consonant with other views of phasic NE, including that it is "driven by decision processes involved with identification of motivationally targeted stimuli [and might] ... support and facilitate task-driven phasic behaviors and thereby promote selective behavioral responding" (Rajkowski et al. 2004; see also Clayton et al. 2004); and that it might "provoke or facilitate dynamic reorganization of target neural networks, permitting rapid behavioral adaptation to changing environmental imperatives" (Bouret \& Sara 2005), so acting as a reset signal. Indeed, there have been other suggestions that NE acts as a form of internal interrupt signal (see Ego-Stengel et al. 2002; David Johnson 2003). By tying phasic NE very precisely to the evolution of knowledge about a particular aspect of the state within a task, our theory takes a further step towards the quantification of this key signal.

As one might expect for an interrupt signal, NE activation is subject to extensive topdown and bottom-up influences (Aston-Jones et al. 1986; 1991; Arnsten \& Goldman-Rakic 1984; Van Bockstaele et al. 1996; 1999a;b; Jodo et al. 1998; Bouret et al. 2003). Bottomup influences can mediate interrupts arising from grossly unexpected contingencies such as critical threats and stressors. Top-down influences, putatively coming from frontal cortical areas such as the anterior cingulate cortex, have potent top-down projections to the locus coeruleus (Jodo et al. 1998; Sara \& Hervé-Minnvielle 1995; see also Aston-Jones \& Cohen 2005a;b), and could implement more subtly contingent interruptability.

We reify our theory in a Bayesian model of the task that generated the data of Figure $1 \mathrm{~B} ; \mathrm{C} ; \mathrm{D}$, and demonstrate that it captures some key aspects of the electrophysiological data not easily explained by previous quantitative models (Brown et al. 2004; Brown et al. 2005).

\section{Results}

A simple discriminative vigilance task for monkeys was used to collect the data in Figure $1 \mathrm{~B}-\mathrm{D} ; \mathrm{F}$. Formally, this involves a sequence of states, starting from the animal pressing a footpedal and fixating a central cue, going through the presentation of a target or non-target stimulus a short time later, eliciting a response or non-response and possibly a reward, and ending with a random inter-trial interval. We assume that the monkey, in becoming facile at the task, learns a (possibly implicit) internal model of these states and the possible transitions among them. Tonic NE (along with many other systems) will likely play a role in directing learning about the task contingencies, as uncertainty about the statistical structure of a task is a critical factor driving learning (Yu \& Dayan 2005). For the remainder of the paper, we assume that the monkey has already learned a correct internal model for the task, and focus on the inferential rather than the learning aspects of stimulus processing.

The task involves some straightforward uncertainties about (a) when the target or distractor will come on following initiation of the trial (due to imperfect interval timimg, Gibbon et al. 1997); and (b) whether it is the target or the distractor that is presented on a particular trial. These uncertainties about states and transitions can be captured by a hidden Markov model (HMM; see e.g., MacKay 2003), which consists of indirectly observed (hidden) states, and probabilistic transitions among those states.

Figure 2A shows a graphical representation of an HMM that characterizes the discrimination task, and which therefore formally quantifies the monkey's knowledge about the timing and nature of the presentation of the stimulus (see Experimental Procedures for more information). There are three states, start (initiated by the fixation) and target and distractor 
A
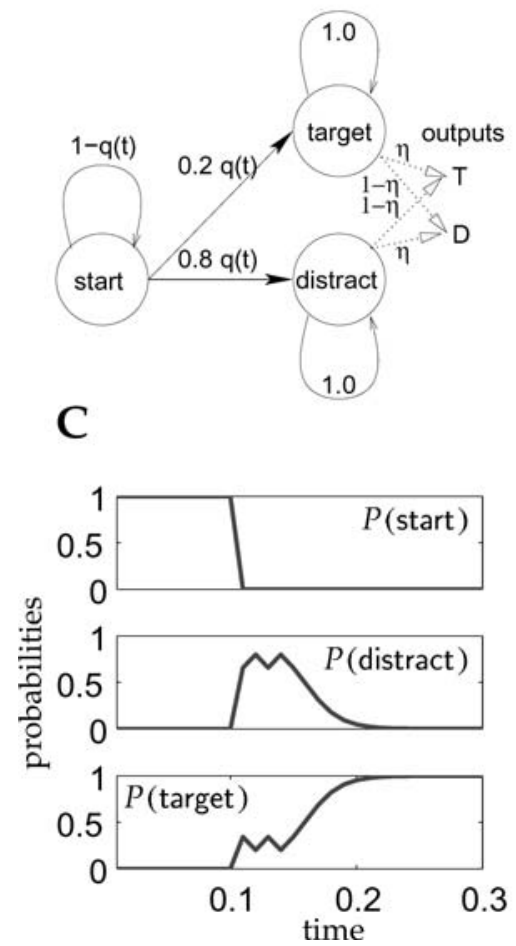

B
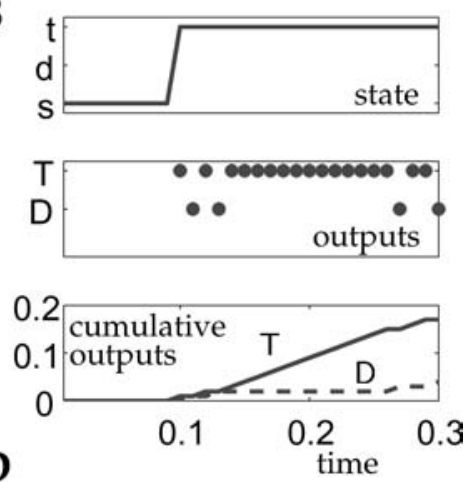

D
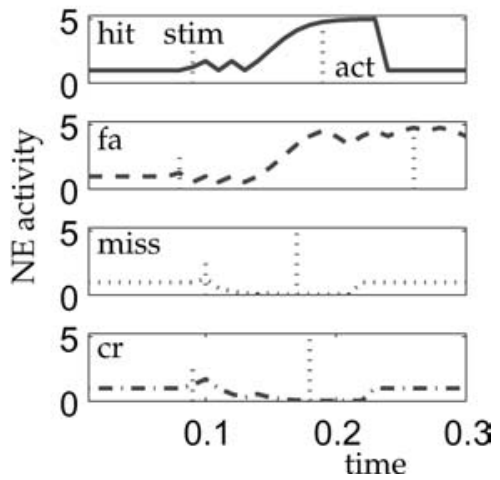

Figure 2. The model. A) The task is viewed as a hidden Markov model (HMM) with transitions from start to either distractor (with probability $p=0.8$ ) or target (with probability $p=0.2$ ). The transitions are structured so the dwell time in start is drawn from a uniform distribution between 5 and 10 timesteps $(q(t)=1 /(11-t)$ for timestep $t \in 5,6, \ldots, 10$ and 0 otherwise); distractor and target are absorbing states. As a crude model of discrimination time, we make each time-step be $10 \mathrm{~ms}$. The only outputs are produced at the absorbing states, and, to model the difficulty of discrimination, both states can produce either of the two possible observables, $\mathrm{T}$ or $\mathrm{D}$ but with probabilities $\eta$ and $1-\eta$ for their 'own' output (T for target, and D for distractor). These outputs of the generative model are inputs to the recognition process that leads to the probabilities shown. The main simulations use $\eta=0.675$; a more difficult task employs $\eta=0.65$. B) One single run with a transition from start to target at timestep 10 (upper). The outputs mildly favor target $(\mathrm{T})$ once the state has changed (middle; bottom), to an extent that is clearer in the cumulative plot (bottom). C) Correct probabilistic inference in the task leads to the probabilities for the three states as shown. The distractor's initial advantage arises from a base rate effect, since it is the more likely transition. D) Putative NE signal for four trials including one for hit (top; same as in B;C), a false alarm (fa), a miss (miss) and a correct rejection (cr). The NE signal during start arises from assumed uncertainties in timing; the NE signal after the transition (the first vertical dotted line in each plot) reflects the balance of Ts and Ds. The second vertical line represents the point at which the action was taken (for hit and fa), or a decision that it is a distractor trial (miss and cr). 5 steps after this, the NE signal is set back to baseline (1).

(corresponding to the presentation of the respective stimuli). Each trial begins with fixation and therefore starts in the start state, and transits into either target or distractor after a variable amount of time. The distribution of the variable delay period, over different trials, is determined by the probability of the trial appearing to persist in the start state (self-transitions) for different amounts of time owing to imprecise interval timing (Gibbon et al. 1997). In general, stochastic timing could be built into the experimental design directly. The variable $q(t)$ specifies the temporal hazard function controlling the distribution of (perceived) stimulus onset. 
The relative frequency of target and distractor depends on the relative transition probabilities of start into target versus into distractor. The numbers over the transition arrows indicate the transition probabilities-notably the four times greater probability of transition to distractor, capturing the $80 \%$ frequency of distractors and $20 \%$ frequency of targets in the task. The HMM is a generative model for the task, i.e., the internal model the animal (at least implicitly) has about the task, which we assume to reflect the task's statistical structure veridically.

Finally, we assume that the whole trial starts with fixation, so there is no observable output during the start state. Since the monkeys show errors that depend on the confusability of the target and distractor stimuli, the observations emitted at target and distractor must be at least partially ambiguous. We model this by having two possible observations, one (T) a more likely product of target; the other (D) of distractor. The probabilities with which the observations are emitted at each state specify how confusable target and distractor are, and consequently how difficult the discrimination is (see Experimental Procedures).

Figure $2 \mathrm{~B}$ shows a sample run from the model. The top plot shows the state at each time- - this remained in start for just a few steps, made a transition to target. The middle plot shows the observations generated at each time-even though the transition was to target, both $\mathrm{T}$ and $\mathrm{D}$ symbols are produced because of the confusability. The bottom plot shows the cumulative observations of $\mathrm{T}$ and $\mathrm{D}$; these observations provide the sole inputs with which the animal must make a decision about the current stimulus.

In order to maximize reward, the goal for the animal is to detect correctly the transition out of start into target or distractor and then respond or withhold correspondingly. That is, the subject must perform probabilistic inference, using the confusable observations as data.

Figure 2C shows the result of exact probabilistic inference in the HMM for the observations arising in the example run shown in Figure 2B. The mathematical details can be found in Experimental Procedures; here, we provide an intuitive description. The upper trace in Figure 2C shows the evolution of the posterior probability that the state is in start over the timecourse of a trial; the middle and lower traces show respectively the posterior probabilities of target and distractor. These latter both start out at 0 , since, under the model, the animal knows that it begins in start. As soon as one of the target or distractor outputs ( $\mathrm{T}$ or $\mathrm{D})$ is shown, the probability of start becomes 0 , since neither output is possible from this state. There then ensues an inferential battle between target and distractor, with the latter having the initial advantage because its prior probability is $80 \%$. However, the probability of target gets close to 1 (correctly in this example) after around 20 timesteps $(200 \mathrm{~ms})$. Exact inference is easy in this task, since there are only two possible transitions out of start; in more complex tasks, such as the one we considered in the model of tonic NE and ACh (Yu \& Dayan 2005), exact inference imposes computational demands that are too severe for ready biological implementation, making approximations mandatory.

We treat the monkey as acting in favor of target or distractor when the probabilities of transitions into these states are sufficiently high (when they cross a fixed threshold, see Experimental Procedures). This is related to the sequential probability ratio test (SPRT; Wald 1947), which has received wide attention in psychological (see Ratcliff \& Smith 2004) and neuroscientific (see Gold \& Shadlen 2002) investigations, and which also underlies the gain-scheduling model of Brown et al. $(2004 ; 2005)$. In order to capture behavioral errors more accurately, we also include a tiny chance that the animal reacts prematurely on any given trial: the animal releases the bar independent of the posterior probability, leading to a correct response on a target trial, but more often to an error on the more common distractor trial. 

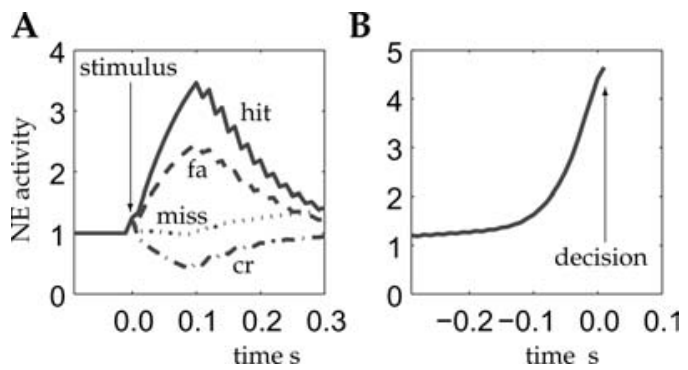

Figure 3. NE activity. A) NE activity locked to the delivery of the first stimulus i.e., the transition out of start for the four types of trial). B) NE activity response-locked to the decision to act, just for hit and fa trials (the two curves lie almost exactly on top of each other). Note the difference in scale between the two figures.

Figure 2C shows the raw material out of which our model of phasic NE is built; in this context, we propose that NE reports on the ratio of posterior probability of a target being present to its prior probability. That is, it signals $P$ (target $\left.\mid x_{1}, \ldots, x_{t}\right) / P$ (target), where $x_{1}, \ldots, x_{t}$ are all the observations up to, and including, time $t$. This identification is based on the following three key assumptions. First, phasic NE reports unexpected uncertainty about the current state in the model, and not about the overall task (which is the role for tonic NE in Yu \& Dayan 2005). Second, there is a default state sequence: start $\rightarrow$ distractor, against which NE measures uncertainty. We discuss this assumption at length below. The uncertainty can have a temporal aspect, for the transition out of start, and a structural one, as when uncertainty builds up in distractor in cases (see Figure 2C) in which the target is successfully detected. The third assumption is that the uncertainty is measured explicitly by divisive comparison with the prior probability in the model about the current state. This assumption is based on suggestions in the data of Alexinsky et al. (1990); Rajkowski et al. (2002), that making targets more probable reduces NE activity. Since all the published experiments use only one or two possible frequencies for the target, it is not currently possible to assess completely how NE activation scales with prior uncertainty in a graded fashion. However, this is a less critical aspect of our theory.

Figure 2D (top) shows the model NE signal in this same case. The substantial response during the presentation of the specific outputs comes as the subject is ever more compelled to consider the state as having changed to target instead of distractor and thereby react appropriately. When the probability reaches a threshold level (0.95), a response is triggered (Gold \& Shadlen 2002; Ratcliff 2004), and, after a delay of $50 \mathrm{~ms}$, the NE signal is set back to baseline (1). This particular form of NE reset is chosen for ease. The data in Figure 1D hint at a rather richer time-course to the $\mathrm{NE}$ activity following the triggering of a response, which may arise from the intrinsic dynamics (for instance, involving autoreceptors) associated with the activity of the NE neurons. Note that the precise form of the mapping from unexpected uncertainty to NE spikes is rather arbitrary. In particular, there may be a strong non-linearity, or even a threshold on the uncertainty, below which there is little activity. In the absence of clear countermanding data, we assume a linear mapping for simplicity.

The remaining rows of Figure 2D show NE activity in single trials of the other three classes of response (fa: false alarm, miss, and cr: correct rejection). Figure 3A, a main result of this paper, shows the averages of these signals across trials of all classes, time-locked to the start of the stimulus. These traces should be compared with those in Figure 1D. The small response around the onset of the stimulus (which is present in all the cases in Figure 2D) arises from the uncertainty about the timing of the transition out of the start state caused 
by the variability of interval timing (see Gibbon et al. 1997). This timing uncertainty will affect the nature of the model that the subjects can build as to the statistics of the time of the appearance of the stimulus after the fixation point, and also how surprised they are on each trial when the stimulus actually arrives. These considerations suggest that there will only be a small NE response unless the stimulus is very unusually early or late-a condition for which there are currently no neurophysiological data. In the absence of such data, we model it simply by giving it a small value.

The basic form of the rise of the main signal in the model (Figure 3A) is broadly similar to that in the data (Figure 1D); as we have argued, the fall is rather arbitrary. For simplicity, we do not model the additional variability in response time that arises not from the inferential or decision process, but from other sources such as variability in the execution of the motor command. Figure 3B shows the average signal locked to the time of reaction (for both hit and false alarm trials) rather than stimulus onset. As also in the data (Figure 1E), responselocked activities are much more tightly clustered. That the traces for hits and false alarms lie on top of each other in the response-triggered plot (Figure 3B) but not the stimulus triggered plot (Figure 3A) suggests that the reaction time is more variable for the false alarms. Since the decay of the signal following a response is unconstrained, the trace terminates when the response is determined, usually when the probability of target reaches threshold, but also sometimes when there is a premature response.

Figure 4 shows some additional features of the NE signal in this case. Figure 4A compares the effect on the NE signal in hit trials of making the discrimination between target and distractor more or less difficult in the model (upper) and in the data (lower; Rajkowski et al. 2004). As in the data, the stimulus-locked NE signal is broader for the more difficult case, since information has to build up over a longer period. Also as in the data, correct rejections are much less affected than hits. Figure 4B shows response locked NE. Certain details in the NE response are not modeled here, for instance the reduction in the delay between the peak of the NE response and the behavioral response in the difficult condition (Rajkowski et al. 2002). One possibility is that the longer times for integrating information about the stimuli makes for greater motor preparedness, and therefore faster responding following the sensory-based decision.

\section{Discussion}

The present model of the phasic activity of NE cells is a complementary extension of our previous model of tonic aspects of this neuromodulator. The key difference is that unexpected uncertainty is now about the state within a task rather than about the characterization of the task as a whole. By formalizing the drive behind the phasic NE signal that resets or interrupts ongoing processing (see also David Johnson 2003; Aston-Jones \& Cohen 2005a;b; Bouret \& Sara 2005), this theory can account for a broad range of data.

For the task modeled here, the specific effect of the NE-mediated interrupt is to arouse the animal from default inaction to release a continuously pressed bar in response to an unexpected target stimulus. More general roles for such an interrupt include organizing more general aspects of behavioral responding as well as ensuring that top-down influences on sensory processing associated with the default, current state are immediately nullified once their statistical foundations have been undermined. These effects are generally very wideranging, affecting sensory, motor, cognitive and affective systems. NE's fast responses and diffuse projections make it particularly suitable for signaling the detection of such unexpected state changes. The actual maintenance of the contextual model, and the computation of the 
A

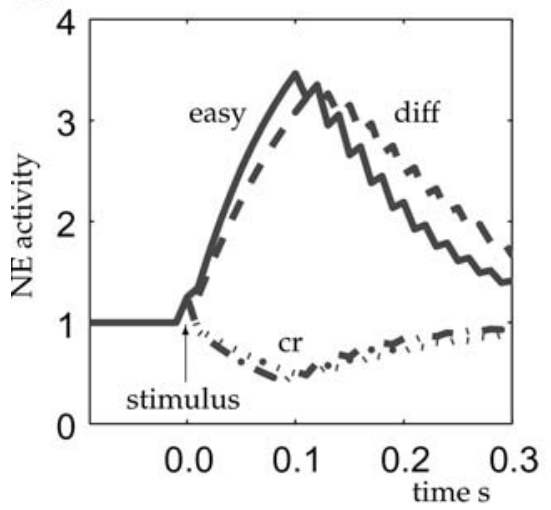

B
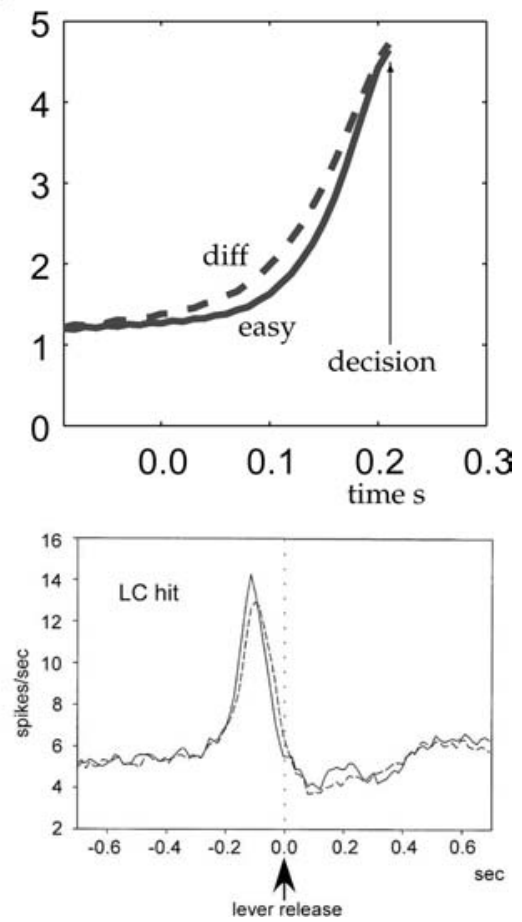

Figure 4. NE and discrimination difficulty. If the output distributions for target and distractor are made more similar by reducing $\eta$ (see Figure 2A), the NE signal gets broader and lower, as in the data. A) Upper plot shows average $\mathrm{NE}$ activity locked to the stimulus for easy (solid, hits; dot-dashed, correct rejections for $\eta=0.675$, as in Figures 2 and 3) and difficult (dashed, hits; dotted, correct rejections for $\eta=0.65$ ) tasks, for comparison with the average activity of NE cells in an analogous experimental manipulation (lower). B) Response-locked plots for hits for easy (solid) and difficult (dashed) conditions from the model (upper) and the data (lower). Data taken from Rajkowski et al. (2004).

posterior probabilities, are likely to be subserved by the prefrontal cortex (Braver et al. 1999), and also the anterior cingulate cortex, which has been shown to be activated by violations of expectations (Luu \& Pederson 2004). Both these neural structures exert a powerful influence over the locus coeruleus, as indeed do subcortical structures, which could occasion interrupts based on more primitively assessed unexpected events.

In the task we modeled, the consequence of a switch in the state from the default distractor to target is a behavioral response. One might suggest that NE is therefore really only an intimate part of the response system, rather than having any wider role in inference, such as unravelling sensory inferences that have been based on incorrect assumptions of a default state. Indeed, nearly all the results we know of, including those on completely different tasks, such as noradrenergic influences in the countermanding saccade task (Chamberlain et al. 2006), do not study the effect on ongoing sensory inferences. Nevertheless, four pieces of circumstantial evidence argue against this narrower view. First, the small, but significant, enhancement in NE activity very shortly after the stimulus is presented (the asterisk in Figure 1D) is not accompanied by a response. It is associated with a change in state (from no stimulus to stimulus) and, according to our model, arises because of the subjective temporal uncertainty at the onset of the stimulus. The precise form of this timing-related uncertainty 
deserves closer scrutiny. For instance, if the monkeys were to build a more sophisticated model of the timing of the transition out of start, then the NE signal might be activated even before the onset of the stimulus, whenever it arrives, and particularly if it is unexpectedly late, in a manner similar to dopamine-mediated reward prediction errors (Schultz et al. 1997). We would also expect this transition to result in a different NE signature if either there were explicit, experimenter-determined temporal uncertainty in the delivery of the stimulus, or if the start state were to generate sensory inputs that could be confused with those generated during target and distractor.

The second and third issues for the motor response theory are that substantial motor responses can happen without NE activity (as is explicitly discussed by Bouret \& Sara 2005), and stimulus-specific LC activity can occur without explicit motor effects (for instance in the reversal conditions in Aston-Jones et al. 1997). The final issue is that the magnitude of LC responses decreases as a function of the target probability (Alexinsky et al. 1990; Aston-Jones et al. 1994; Rajkowski et al. 2002), while the behavioral response is maintained across these conditions.

None of these individual arguments is completely definitive. Further experiments could help tease unexpectedness apart from behavioral responding. For instance, consider a task with second-order contingencies. Suppose that observations at one time point in the task specify the state at that time, but the behavioral consequence is not specified until later. According to our account, NE activity would be tied to the observation-induced state change, independent of behavior. The small activation when the stimulus is presented (evidence in Figure 1D) is effectively an example of this. Careful experimental design is necessary to avoid the default sequence of states in the learned model from involving behavioral quiescence, with unexpected state changes engendering behavioral output, since the NE will indeed appear to be tied to actions. Perhaps the most direct test would be to measure the noradrenergic sensitivity of persistent or accumulating activity associated with ongoing sensory inferences, for instance in random-dot direction discrimination tasks patterned after Roitman \& Shadlen (2002).

The early NE "bump" and the decrease in activity as a function of probability are also key issues for a most interesting optimal gain-scheduling model (Brown et al. 2004; 2005; AstonJones \& Cohen 2005a;b) of the Eriksen flanker discrimination task (Clayton et al. 2004) that requires two different active responses to two types of stimuli (by contrast with the go/no-go nature of the task modeled here). That model formalized earlier, somewhat more heuristic, suggestions (Usher et al. 1999) as to how NE may be an integral part of an optimal decision strategy. Brown et al. $(2004 ; 2005)$ proposed that NE controls the gain in competitive decision-making networks such as the drift diffusion networks discussed by Smith \& Ratcliff (2004) and the leaky accumulator networks of Usher \& McClelland (2001). One aspect of this view is that the presentation of the stimulus implies that the statistical structure of the input has changed (gaining a non-zero signal-to-noise ratio). Consequently, optimal processing would require the inputs to be integrated differently (relied upon more). NE can effect this difference by changing the gain in decision making networks associated with the input integration. It is also suggested that a more extreme change in the gain, destabilizing the competitive networks through explosive symmetry breaking, can be used to freeze or lock-in any small difference in the competing activities.

The idea that NE can signal the change in the input statistics occasioned by the (temporally variable) occurrence of the target is highly appealing. Indeed, this theory makes an important link to sequential statistical analysis (Wald 1947; Barnard 1946), raising issues about things like thresholds for deciding target and distractor, that should be important foci of future work 
in the context of our model. Our form of normative inference in an HMM is very closely related to these theories, notably in the case that there are only two possible states (here, target and distractor). However, in the context of the current go/no-go task, the statistics of the input change when either the target or the distractor appears, and so the preference of NE neurons for responding to the target alone is unaccountable. It is possible that the early small bump in the activity, which arises for both classes of stimuli, could support this argument, but then the cause for the later and much larger response is not so clear. The effect of forcing the decision-making network to become unstable, and therefore enforcing a speeded decision, is like a reset signal. However, since the statistical optimality of integration is effectively abandoned in the unstable regime, the normative rationale behind the optimal decision-making argument is diminished. Further, there would be no reason under this account why this signal should decrease as the target becomes more probable (Alexinsky et al. 1990; Rajkowski et al. 2002).

The Eriksen flanker task underpinning the gain-scheduling model (Clayton et al. 2004) involves two equally (in)frequent targets, which both impose requirements for action. In this task, NE is released to both possible targets. This would be captured under our account by a different internal model for the states, for instance with transitions to either stimulus state constituting unexpected transitions away from a default start state. A similar explanation might underlie the observation that noradrenergic neurons recorded in the rat locus coeruleus are activated to a rewarded odor stimulus that appears on $50 \%$ of the trials. Indeed, our theory puts onto center stage the exact nature of the subjects' internal characterization of a task. For the main set of experimental data modeled in this work, this internal representation is proposed to be a hidden Markov model. Model building of this sort (either explicit or implicit), its putative instantiation in the prefrontal cortex (Owen 1997), and indeed the effects on learning of tonic NE and acetylcholine (Yu \& Dayan 2005), are topics of active interest. Model building also figures prominently in goal-directed behavior (e.g., Daw et al. 2005a;b).

The distractor task involves only two states, and so makes the likelihood computation straightforward. Given more alternatives, it would seem less reasonable to suggest that the posterior probability of each could be separately maintained. Exactly the same issue arises in many other cases for probabilistic inference; in Yu \& Dayan (2005), we suggested that the posterior probability of only a single, contextually-dependent (between-task) default state be calculated fully, with the probability that this state is wrong being represented by tonic NE (and being shared equally among the non-dominant state. We would make exactly the same hypothesis for phasic NE, bar the normalization by the prior probability. Unfortunately, we know of no directly relevant data about this issue.

Figure $1 \mathrm{~F}$ shows an additional phenomenon that arises when the monkeys are not even occasionally taxed with difficult discrimination problems: a periodic fluctuation between "phasic" and "tonic" modes (Usher et al. 1999). This effect did not arise in the version of the task in Rajkowski et al. (2004) that we modeled or the Eriksen flanker task (Clayton et al. 2004). In the case of Figure 1F, the overall performance fluctuates dramatically (shown by the changing false alarm rate), in a manner that is tightly correlated with fluctuations in tonic NE activity. Periods of high tonic activity are correlated with low phasic activation to the targets and high false-alarm rate (data not shown). Aston-Jones, Cohen and their colleagues (Usher et al. 1999; Aston-Jones et al. 2000; Aston-Jones \& Cohen 2005a;b) have suggested that NE regulates the balance between exploration and exploitation, as, in a related formulation, has Doya (2002). The high tonic phase is associated with the former, with monkeys failing to concentrate on the contingencies that lead to their current rewards in 
order to search for stimuli or actions that might be associated with better rewards. Increasing the ease of interruptability to either external cues or internal state changes, could certainly lead to apparently exploratory behavior. However, it is not completely clear how this sort of exploration is being actively determined, since, for instance, the macroscopic fluctuations evident in Figure 1F do not arise in response to any experimental contingency. Given the relationship between phasic and tonic firing, further investigation of these periodic fluctuations and their implications is desirable.

Finally, in our previous model (Yu \& Dayan, 2003; 2005), tonic NE was closely coupled with tonic acetylcholine $(\mathrm{ACh})$, with the latter reporting expected rather than unexpected uncertainty. The account of ACh should transfer somewhat directly into the short-term contingencies within a task-we might expect it to be involved in reporting on aspects of known variability associated with each state, including each distinct stimulus state as well as the no-stimulus state. As such, this ACh signal might be expected to be relatively more tonic than NE (an effect that is also apparent in our previous work on more tonic interactions between ACh and NE (e.g., Figure 2 of Yu \& Dayan 2003). One attractive target for an account along these lines is the sustained attention task studied by Sarter and colleagues (see Sarter et al. 2001), which involves temporal uncertainty (Yu et al. 2005). Performance in this task is exquisitely sensitive to cholinergic manipulation, but unaffected by gross noradrenergic manipulation (McGaughy et al. 1997). We may expect there to be interesting part-opponent and part-synergistic interactions between the phasic aspects of the two neuromodulators.

\section{Methods}

Figure 2A illustrates a simple hidden Markov generative model (HMM) of the discrimination task in Figure 1B-E. The start state models the condition established when the monkey fixates the light and initiates a trial. Following a variable delay, either the target or the distractor is presented, and the monkey must respond appropriately (release a continuously pressed bar for target and continue pressing for distractor). As a very simple model, the transition out of start is uniformly distributed between timesteps 6 and 10 ( 0 probability otherwise), implemented by a time-varying transition function $q_{t}$ for this node (equivalent to a discrete semi-Markov state):

$$
P\left(s_{t} \mid s_{t-1}=\text { start }\right)= \begin{cases}1-q_{t} & s_{t}=\text { start } \\ 0.8 q_{t} & s_{t}=\text { distractor } \\ 0.2 q_{t} & s_{t}=\text { target }\end{cases}
$$

where $q_{t}=1 /(11-t)$ for $(6 \leq t \leq 10)$ and $q_{t}=0$ otherwise. Each timestep in the model is about $10 \mathrm{~ms}$, which produces the range of experimentally observed discrimination times. The time in start is not taken to match the experimental contingency. The target and distractor states are assumed to be absorbing states (with self-transition probability 1). Given that a transition out of start (into either target or distractor) takes place, the probability is 0.2 for entering target and 0.8 for start, as in the actual task. In addition, it is assumed that the node start does not emit observations, while target emits $x_{t}=\mathrm{T}$ with probability $\eta>0.5$ and $\mathrm{D}$ with probability $1-\eta$, and distractor emits $x_{t}=\mathrm{D}$ with probability $\eta$ and T with probability $1-\eta$.

The transition out of start is evident as soon as the first D or T is observed, while the magnitude of $\eta$ controls the "confusability" of the target and distractor states. Figure $2 \mathrm{~B}$ shows a typical run from this generative model. The transition into target happens on step 10 
(top), and the outputs generated are a mixture of T and D (middle), with an overall prevalence of $\mathrm{T}$ (bottom).

Exact inference on this model can be performed in a manner similar to the forward pass in a standard HMM:

$$
P\left(s_{t} \mid x_{1}, \ldots, x_{t}\right) \propto p\left(x_{t} \mid s_{t}\right) \sum_{s_{t-1}} P\left(s_{t} \mid s_{t-1}\right) P\left(s_{t-1} \mid x_{1}, \ldots, x_{t-1}\right)
$$

for $s_{t}$ set to, start, distractor and target.

Because start does not produce outputs, as soon as the first $\mathrm{T}$ is observed, the probability of start plummets to 0 . There then ensues an inferential battle between target and distractor, with the latter having the initial advantage, since its prior probability is $80 \%$.

Because of the preponderance of transitions to distractor over target, the distractor state can be thought of as the reference or default state. Evidence against that default state is a form of unexpected uncertainty within a task, and we propose that phasic NE reports this uncertainty. More specifically, NE signals $P$ (target $\left.\mid x_{1}, \ldots, x_{t}\right) / P$ (target), where $P$ (target) $=0.2$ is the prior probability of observing a target trial. We assume that a target-response is initiated when $P$ (target $\left.\mid x_{1}, \ldots, x_{t}\right)$ exceeds 0.95 , or equivalently, when the NE signal exceeds $0.95 / P$ (target) $=0.95 / 0.2=4.75$. This implies the following intuitive relationship: the smaller the probability of the (non-default) state target the greater the NE-mediated "surprise" signal has to be in order to convince the inferential system that an anomalous stimulus has been observed. We also assume that if the posterior probability of target reaches 0.01 , then the trial ends with no action (choosing distractor, which results in either a cr or a miss). The asymmetry in the thresholds arises from the asymmetry in the response contingencies of the task. Further, to model non-inferential errors, we assume that there is probability of 0.0005 per timestep of releasing the bar after the transition out of start. Once a decision is reached, the NE signal carries on accumulating for a response delay of 5 timesteps before being set back to baseline (1, for equal prior and posterior). The apparent jagged staircase in the descent of the NE signal arise because there are only two possible observations, which exactly cancel each other out in the likelihood. They would not be present if, for instance, there was the random possibility of not observing anything at all on a time-step despite being in distractor or target.

For the easy task, we set $\eta=0.675$; for the "difficult" condition of Figure $4, \eta=0.65$. For the easy task, there were $19 \%$ hits, $1.5 \%$ false alarms, $1 \%$ misses and $77 \%$ correct rejections. For the difficult task, the main difference was an increase in the number of misses to $1.5 \%$, largely at the expense of hits.

Note that the precise form of the mapping from unexpected uncertainty to NE spikes is rather arbitrary. In particular, there may be a strong non-linearity, such as a thresholded response profile. For simplicity, we assume a linear mapping between the two.

The NE activity during the start state is also rather arbitrary. Activity is at baseline before the stimulus comes on, since prior and posterior match when there is no explicit information from the world. When the stimulus comes on, the divisive normalization makes the activity go above baseline because, although the transition was expected, its occurrence was not predicted with perfect precision. The magnitude of this activity depends on the precision of the model of the time of the transition; and the uncertainty in the interval timer. We set it to a small super-baseline level to match the data. 


\section{Acknowledgements}

We are particularly grateful to Sebastien Bouret, Eric Shea-Brown, Gary Aston-Jones, Jonathan Cohen, Mark Gilzenrat, Phil Holmes and Susan Sara for sharing ideas and data before their publication, and to Peter Latham, Sam McClure and Ben Seymour for their comments on the work and the paper. Two reviewers provided most helpful comments and advice. This study was funded by the Gatsby Charitable Foundation, the EU BIBA project and the ACI Neurosciences Intégratives et Computationnelles of the French Ministry of Research.

\section{References}

Alexinsky T, Aston-Jones G, Rajkowski J, Revay RS. 1990. Physiological correlates of adaptive behavior in a visual discrimination task in monkeys. Society for Neuroscience Abstracts 16:164.

Arnsten AF, Goldman-Rakic PS. 1984. Selective prefrontal cortical projections to the region of the locus coeruleus and raphe nuclei in the rhesus monkey. Brain Res 306:9-18.

Aston-Jones G, Bloom FE. 1981. Norepinephrine-containing locus coeruleus neurons in behaving rats exhibit pronounced responses to non-noxious environmental stimuli. J Neurosci 1:887-900.

Aston-Jones G, Cohen JD. 2005a. An integrative theory of locus coeruleus-norepinephrine function: Adaptive gain and optimal performance. Ann Rev Neurosci 28:403-450.

Aston-Jones G, Cohen JD. 2005b. Adaptive gain and the role of the locus coeruleus-norepinephrine system in optimal performance. J Comp Neurology 493:99-110.

Aston-Jones G, Ennis M, Pieribone VA, Nickell WT, Shipley MT. 1986. The brain nucleus locus coeruleus: Restricted afferent control of a broad efferent network. Science 234:734-737.

Aston-Jones G, Rajkowski J, Cohen J. 2000. Locus coeruleus and regulation of behavioral flexibility and attention. Prog Brain Res 126:165-182.

Aston-Jones G, Rajkowski J, Kubiak P. 1997. Conditioned responses of monkey locus coeruleus neurons anticipate Acquisition of discriminative behavior in a vigilance task. Neurosci 80:697-715.

Aston-Jones G, Rajkowski J, Kubiak P, Alexinsky T. 1994. Locus coeruleus neurons in monkey are selectively activated by attended cues in a vigilance task. J Neurosci 14:4467-4480.

Aston-Jones G, Shipley MT, Chouvet G, Ennis M, Van Bockstaele EJ, Pieribone V, Shiekhattar R, Akaoka H, Drolet G, Astier B, Charlety P, Valentino R, Williams JT. 1991. Afferent regulation of locus coeruleus neurons: Anatomy, physiology and pharmacology. Prog Brain Res 88:47-75.

Barnard GA. 1946. Sequential tests in industrial statistics. J Royal Statistical Soc Supplement 8:1-26.

Berridge CW, Waterhouse BD. 2003. The locus coeruleus-noradrenergic system: modulation of behavioral state and state-dependent cognitive processes. Brain Res Rev 42:33-84.

Bouret S, Duvel A, Onat S, Sara SJ. 2003. Phasic activation of locus coeruleus neurons by the central nucleus of the amygdala. J Neurosci 23:3491-3497.

Bouret S, Sara SJ. 2004. Reward expectation, orientation of attention and locus coeruleus-medial frontal cortex interplay during learning. Eur J Neurosci 20:791-802.

Braver TS, Barch DM, Cohen JD. 1999. Cognition and control in schizophrenia: a computational model of dopamine and prefrontal function. Biol Psychiatry 46:312-328.

Brown E, Gao J, Holmes P, Bogacz R, Gilzenrat M, Cohen JD. 2005. Simple neural networks that optimize decisions. Int J Bifurcation Chaos 15:803-826.

Brown ET, Gilzenrat MS, Cohen JD. 2004. The locus coeruleus, adaptive gain, and the optimization of simple decision tasks. Technical Report 04-02. Center for the Study of Brain, Mind and Behavior, Princeton University.

Chamberlain SR, Muller U, Blackwell AD, Clark L, Robbins TW, Sahakian BJ. 2006. Neurochemical modulation of response inhibition and probabilistic learning in humans. Science 311:861-863.

Clark CR, Geffen GM, Geffen LB. 1989. Catecholamines and the covert orientation of attention in humans. Neuropsychologia 27:131-139.

Clayton EC, Rajkowski J, Cohen JD, Aston-Jones G. 2004. Phasic activation of monkey locus coeruleus neurons by simple decisions in a forced-choice task. J Neurosci 24:9914-9920.

Curet O, Dennis T, Scatton B. 1987. Evidence for the involvement of presynaptic alpha-2 adrenoceptors in the regulation of norepinephrine metabolism in the rat brain. J Pharmacology Exp Therapeutics. 240:327-336.

David Johnson J. 2003. Noradrenergic control of cognition: global attenuation and an interrupt function. Med Hypotheses 60:689-692. 
Daw ND, Courville AC, Touretzky DS. 2003. Timing and partial observability in the dopamine system. Advances in Neural Information Processing Systems 15:99-106.

Daw ND, Kakade S, Dayan P. 2002. Opponent interactions between serotonin and dopamine. Neural Networks 15:603-616.

Daw ND, Niv Y, Dayan P. 2005a. Actions, policies, values, and the basal ganglia. To appear in Bezard, editor. Recent breakthroughs in basal ganglia research. New York, NY: Nova Science Publishers.

Daw ND, Niv Y, Dayan P. 2005a. Actions, policies, values, and the basal ganglia. To appear in Bezard, editor. Recent breakthroughs in basal ganglia research. New York, NY: Nova Science Publishers.

Daw ND, Niv Y, Dayan P. 2005b. Uncertainty-based competition between pre-frontal and dorsolateral striatal systems for behavioral control. Nature Neurosci 8:1704-1711.

Dayan P, Yu A. 2001. ACh, uncertainty, and cortical inference. Advances in Neural Information Processing Systems 14:189-196.

Dayan P, Yu AJ. 2003. Uncertainty and learning. IETE J Research 49:171-182.

Devauges V, Sara SJ. 1990. Activation of the noradrenergic system facilitates an attentional shift in the rat. Behavioral Brain Res 39:19-28.

Doya K. 2002. Metalearning and neuromodulation. Neural Networks 15:495-506.

Ego-Stengel V, Bringuier V, Shulz DE. 2002. Noradrenergic modulation of functional selectivity in the cat visual cortex: An in vivo extracellular and intracellular study. Neurosci 111:275-289.

Foote SL, Aston-Jones G, Bloom EE. 1980. Impulse activity of locus coeruleus neurons in awake rats and monkeys is a function of sensory stimulation and arousal. Proc Nat Acad Sciences 77:3033-3037.

Foote SL, Bloom FE, Aston-Jones G. 1983. Nucleus locus coeruleus: New evidence of anatomical and physiological specificity. Physiological Rev 63:844-914.

Foote SL, Freedman R, Oliver AP. 1975. Effects of putative neurotransmitters on neuronal activity in monkey auditory cortex. Brain Res 86:229-242.

Freedman R, Foote SL, Bloom FE. 1975. Histochemical characterization of a neocortical projection of the nucleus locus coeruleus in the squirrel monkey. J Comparative Neurology 164:209-231.

Garver DL, Sladek JR, Jr. 1976. Monoamine distribution in primate brain. II. Brain stem catecholaminergic pathways in Macaca speciosa (arctoides). Brain Res 103:176-182.

Gibbon J, Malapani C, Dale CL, Gallistel C. 1997. Toward a neurobiology of temporal cognition: advances and challenges. Curr Opin Neurobiology 7:170-184.

Gold J, Shadlen M. 2002. Banbarismus and the brain: Decoding the relationship between sensory stimuli, decisions and reward. Neuron 36:299-308.

Hasselmo ME, Linster C, Patil M, Ma D, Cekic M. 1997. Noradrenergic suppression of synaptic transmission may influence cortical signal-to-noise ratio. J Neurophysiology 77:3326-3339.

Jodo E, Chiang C, Aston-Jones G. 1998. Potent excitatory influence of prefrontal cortex activity on noradrenergic locus coeruleus neurons. Neurosci 83:63-79.

Jouvet M. 1969. Biogenic amines and the states of sleep. Science 163:32-41.

Luu P, Pederson SM. 2004. The anterior cingulate cortex: Regulating actions in context. In Posner MI, editor. Cognitive neuroscience of attention. New York: Guilford Publications, Inc.

MacKay DJC. 2003. Information theory, inference and learning algorithm. Cambridge, UK: Cambridge University Press.

Mantz J, Milla C, Glowinski J, Thierry AM. 1988. Differential effects of ascending neurons containing dopamine and noradrenaline in the control of spontaneous activity and of evoked responses in the rat prefrontal cortex. Neurosci 27:517-526.

Marder E, Thirumalai V. 2002. Cellular, synaptic and network effects of neuromodulation. Neural Networks 15:479-493.

McGaughy J, Sandstrom M, Ruland S, Bruno JP, Sarter M. 1997. Lack of effects of lesions of the dorsal noradrenergic bundle on behavioral vigilance. Behavioral Neuroscience 111:646-652.

Montague PR, Dayan P, Sejnowski TJ. 1996. A framework for mesencephalic dopamine systems based on predictive Hebbian learning. J Neurosci 16:1936-1947.

Nicola SM, Woodward Hopf E, Hjelmstad GO. 2004. Contrast enhancement: A physiological effect of striatal dopamine? Cell Tissue Res 318:93-106.

Owen AM. 1997. Cognitive planning in humans: Neuropsychological, neuroanatomical and neuropharmacological perspectives. Progress in Neurobiology 53:431-450.

Rajkowski J, Majczynski H, Clayton E, Aston-Jones G. 2004. Activation of monkey locus coeruleus neurons varies with difficulty and performance in a target detection task. J Neurophysiology 92:361-371.

Rajkowski J, Majczynski H, Clayton E, Cohen JD, Aston-Jones G. 2002. Phasic activation of monkey locus coeruleus (LC) neurons with recognition of motivationally relevant stimuli. Society for Neuroscience 86:10. 
Ratcliff R, Smith PL. 2004. A comparison of sequential sampling models for two-choice reaction time. Psychological Rev 111:333-367.

Roitman JD, Shadlen MN. 2002. Response of neurons in the lateral intraparietal area during a combined visual discrimination reaction time task. J Neurosci 22:9475-9489.

Rolls ET, Thorpe SJ, Boytim M, Szabo I, Perrett DI. 1984. Responses of striatal neurons in the behaving monkey. 3. Effects of iontophoretically applied dopamine on normal responsiveness. Neurosci 12:1201-1212.

Sara SJ, Hervé-Minvielle. 1995. Inhibitory influence of frontal cortex on locus coeruleus neurons. Proc Natl Acad Sci USA, 92:6032-6036.

Sara SJ, Segal M. 1991. Plasticity of sensory responses of locus coeruleus neurons in the behaving rat: implications for cognition. Prog Brain Res 88:571-585.

Sarter M, Givens B, Bruno JP. 2001. The cognitive neuroscience of sustained attention: Where top-down meets bottom-up. Brain Res Rev 35:146-160.

Servan-Schreiber D, Printz H, Cohen JD. 1990. A network model of catecholamine effects: Gain, signal-to-noise ratio, and behavior. Science. 249:892-895.

Smith PL, Ratcliff R. 2004. Psychology and neurobiology of simple decisions. Trends Neurosci 27:161-168.

Usher M, Cohen JD, Servan-Schreiber D, Rajkowski J, Aston-Jones G. 1999. The role of locus coeruleus in the regulation of cognitive performance. Science 283:549-554.

Usher M, McClelland JL. 2001. The time course of perceptual choice: The leaky, competing accumulator model. Psychological Rev 108:550-592.

Van Bockstaele EJ, Chan J, Pickel VM. 1996. Input from central nucleus of the amygdala efferents to pericoerulear dendrites, some of which contain tyrosine hydroxy-lase immunoreactivity. J Neurosci Res 45:289-302.

Van Bockstaele EJ, Peoples J, Telegan P. 1999a. Efferent projections of the nucleus of the solitary tract to peri-locus coeruleus dendrites in rat brain: evidence for a monosynaptic pathway. Journal of Comparative Neurology 412:410-428.

Van Bockstaele EJ, Peoples J, Valentino RJ. 1999b. Anatomic basis for differential regulation of the rostrolateral peri-locus coeruleus region by limbic afferents. Biol Psychiatry 46:1352-1363.

Vankov A, Hervé-Minvielle A, Sara SJ. 1995. Response to novelty and its rapid habituation in locus coeruleus neurons of the freely exploring rat. Eur J Neurosci 7:1180-1187.

Wald A. 1947. Sequential Analysis. New York, NY: John Wiley \& Sons.

Witte EA, Marrocco RT. 1997. Alteration of brain noradrenergic activity in rhesus monkeys affects the alerting component of covert orienting. Psychopharmacology 132:315-323.

Yu AJ, Dayan P. 2002. Acetylcholine in cortical inference. Neural Networks 15:719-730.

Yu AJ, Dayan P. 2003. Expected and unexpected uncertainty. ACh and NE in the neocortex. In Advances in Neural Information Processing Systems 15, Cambridge, MA: MIT Press.

Yu AJ, Dayan P. 2005. Uncertainty, neuromodulation, and attention. Neuron 46:681-692.

Yu AJ. 2005. ACh and NE: Bayes, Uncertainty, Attention, and Learning. PhD Thesis, Gatsby Computational Neuroscience Unit, UCL, University of London. 